\title{
THE BEHAVIOR OF THE CHERN SCALAR CURVATURE UNDER THE CHERN-RICCI FLOW
}

\author{
MATTHEW GILL AND DANIEL SMITH
}

(Communicated by Lei $\mathrm{Ni}$ )

\begin{abstract}
In this paper we study finite-time singularities in the Chern-Ricci flow. We show that finite-time singularities are characterized by the blow-up of the scalar curvature of the Chern connection.
\end{abstract}

\section{INTRODUCTION}

Let $(M, g, J)$ be a complex manifold such that $J$ is compatible with the Riemannian metric $g$. Associated to $g$, we have the Hermitian 2-form $\omega$ given by $\omega(X, Y)=g(J(X), Y)$ for vector fields $X$ and $Y$. We call a manifold admitting such a structure a Hermitian manifold. Recently a number of geometric flows of Hermitian metrics have been introduced to study the underlying Hermitian structure of complex manifolds (e.g. [2, 8], 6], 7], and [3]). The Chern-Ricci flow was introduced by the first-named author in 2 and further developed by TosattiWeinkove in [8. The Chern-Ricci flow is written

$$
\begin{aligned}
\frac{\partial}{\partial t} \omega & =-\operatorname{Ric}^{C}(\omega), \\
\omega(0) & =\omega_{0}
\end{aligned}
$$

where $\operatorname{Ric}^{C}(\omega)=-\sqrt{-1} \partial \bar{\partial} \log \operatorname{det}(g)$. In other words, $\operatorname{Ric}^{C}(\omega)$ is the curvature of the anti-canonical bundle. Notice that when $\omega$ is a Kähler metric, $\operatorname{Ric}^{C}(\omega)$ coincides with the Ricci curvature of $\omega$. Hence if $\omega_{0}$ is Kähler, then (1.1) is the Kähler-Ricci flow.

The Chern-Ricci flow is a diffusion-reaction equation and therefore one expects the development of singularities in the flow. In fact, explicit examples of singularity development are described by Tosatti-Weinkove in [8, [10, and Tosatti-WeinkoveYang in [9]. Moreover in [8] Tosatti-Weinkove give characterizations of finite-time singularities. To describe these characterizations, first notice that the Chern-Ricci flow (1.1) can be written

$$
\begin{aligned}
\frac{\partial}{\partial t} \omega & =-\operatorname{Ric}^{C}\left(\omega_{0}\right)+\sqrt{-1} \partial \bar{\partial} \theta(t), \\
\omega(0) & =\omega_{0}
\end{aligned}
$$

where $\theta(t)=\log \frac{\operatorname{det}(g(t))}{\operatorname{det}\left(g_{0}\right)}$. Hence the solution $\omega(t)$ can be expressed as

$$
\omega(t)=\alpha_{t}+\sqrt{-1} \partial \bar{\partial} \phi(t),
$$

Received by the editors February 7, 2014 and, in revised form, July 27, 2014. 2010 Mathematics Subject Classification. Primary 53C44, Secondary 53C55.

This research was supported by NSF RTG grant DMS-0838703. 
where $\alpha_{t}=\omega_{0}-t \operatorname{Ric}^{C}\left(\omega_{0}\right)$ and $\phi(t)$ is a function satisfying $\frac{\partial}{\partial t} \phi(t)=\theta(t)$ and $\left.\phi\right|_{t=0}=0$. Let

$$
T=\sup \left\{t \geq 0 \mid \exists \psi \in C^{\infty}(M) \text { with } \alpha_{t}+\sqrt{-1} \partial \bar{\partial} \psi>0\right\} .
$$

It is clear that the flow cannot exist beyond time $T$. It was shown by TosattiWeinkove that the flow (1.1), in fact, exists up to time $T$.

Theorem (Tosatti-Weinkove [8]). There exists a unique maximal solution to the Chern-Ricci flow on $[0, T)$.

In addition, Tosatti-Weinkove give the following description of finite-time singularities on complex surfaces.

Theorem (Tosatti-Weinkove [8]). Let $M$ be a compact complex surface and $\omega_{0}$ a $\partial \bar{\partial}$-closed Hermitian metric. Then the Chern-Ricci flow starting at $\omega_{0}$ exists until either the volume of $M$ goes to zero, or the volume of a curve of negative self-intersection goes to zero.

In this note we give an alternative characterization of finite-time singularities in terms of the blow-up of the scalar curvature. In particular we prove

Theorem 1.1. Let $M$ be a compact complex manifold of complex dimension $n$ and $\omega_{0}$ a Hermitian metric. Then the solution $\omega(t)$ of the Chern-Ricci flow (1.1) exists on the maximal interval $[0, T)$ and either $T=\infty$ or

$$
\limsup _{t \rightarrow T}\left(\sup _{M} R(g(t))\right)=\infty
$$

where $R$ denotes the scalar curvature of the Chern connection.

This is a generalization of Zhang's analogous result for Kähler-Ricci flow [11] and the proof employs similar arguments. Tosatti-Weinkove-Yang have also investigated the Chern scalar curvature along the normalized Chern-Ricci flow in the case of elliptic surfaces (with genus strictly larger than one). In particular, they show that the Chern scalar curvature is bounded above by $C e^{t / 2}$ and below by $-C$ and apply this estimate to show that the flow converges exponentially fast to the base in the Gromov-Hausdorff sense [10].

Now we specialize to the case when $\left(M, g_{0}\right)$ is a compact Gauduchon surface, i.e. the associated $(1,1)$ form $\omega_{0}$ of $g_{0}$ has the property that

$$
\partial \bar{\partial} \omega_{0}=0 .
$$

A theorem of Gauduchon shows that every Hermitian metric on a complex surface is conformal to a unique Gauduchon metric. When the Chern-Ricci flow terminates at a finite time $T$, there are two cases to consider: finite-time collapsing and noncollapsing. If $\operatorname{Vol}(M, g(t)) \rightarrow 0$ as $t \rightarrow T$, we say that the flow is collapsing, otherwise we say it is non-collapsing. Tosatti-Weinkove consider the case of finitetime non-collapsing on compact Gauduchon surfaces and show that there are finitely many $(-1)$ curves on $M$ away from which the flow converges smoothly on compact sets [10.

Using the proof of Theorem 1.1 and its result, we are able to show that the set on which singularities develop on $M$ is equal to the region on which Chern scalar curvature blows up. Collins-Tosatti have an analogous result in the Kähler case [1]. Let $g(t)$ be the solution to the Chern-Ricci flow that is non-collapsing in finite 
time. Let $E$ denote the union of the $(-1)$ curves on $M$ that are collapsing as $t \rightarrow T$. Define the singularity formation set $\Sigma$ to be the complement of all points $x$ in $M$ such that there exists an open neighborhood $U$ of $x$ on which $|\operatorname{Rm}(t)| \leq C$ for all $0 \leq t<T$. Also define the set $\Sigma^{\prime}$ to be the complement of all points $x$ in $M$ such that there exists an open neighborhood $U$ of $x$ on which $|R(t)| \leq C$ for all $0 \leq t<T$. Then we have the following result.

Theorem 1.2. Let $\left(M, g_{0}\right)$ be a compact Gauduchon surface that is non-collapsing in finite time along the Chern-Ricci flow. Then

$$
E=\Sigma=\Sigma^{\prime}
$$

If instead the flow is collapsing in finite time, we have

$$
M=\Sigma=\Sigma^{\prime} .
$$

In the final section we include an explicit example of the Chern scalar curvature blowing up like $C(T-t)^{-1}$ along the Chern-Ricci flow in the case of the Hopf manifold.

\section{Preliminaries}

Let $(M, g, J)$ be a closed Hermitian manifold of complex dimension $n$. The Chern connection is the unique connection $\nabla$ satisfying: $\nabla \omega=0, \nabla J=0$, and $T^{1,1}=0$. In local holomorphic coordinates, $z_{1}, \ldots, z_{n}$, the only non-vanishing components of the Chern connection are

$$
\Gamma_{i j}^{k}=g^{k \bar{l}} \partial_{i} g_{j \bar{l}}
$$

and its conjugate. The curvature of the Chern connection is given locally by

$$
R_{i \bar{j} k}^{l}=-\partial_{\bar{j}} \Gamma_{i k}^{l} \text {. }
$$

Taking a trace of the Chern curvature over the last two components yields the Chern-Ricci curvature, that is,

$$
R_{i \bar{j}}^{C}=g^{k \bar{l}} R_{i \bar{j} k \bar{l}}=-\partial_{i} \partial_{\bar{j}} \log \operatorname{det}(g) .
$$

Interestingly, the parabolic flow of Hermitian metrics defined by Streets-Tian in 7 ] is the trace of the Chern curvature over the first two components plus a term that is quadratic in the torsion of $\nabla$. Finally, the Chern scalar curvature, denoted $R$, is defined as the trace of the Chern-Ricci curvature

$$
R=g^{i \bar{j}} R_{i \bar{j}}^{C}
$$

One of the reasons for the recent success of the Chern-Ricci flow is that, like Kähler-Ricci flow, the Chern-Ricci flow is equivalent to a scalar flow. To see this, first recall from (1.2) that the solution of the Chern-Ricci flow can be written

$$
\omega(t)=\alpha_{t}+\sqrt{-1} \partial \bar{\partial} \phi(t) .
$$

Differentiating (2.4) in time, we have

$$
-\operatorname{Ric}^{C}(t)=\sqrt{-1} \partial \bar{\partial} \log \operatorname{det}\left(g_{0}\right)+\sqrt{-1} \partial \bar{\partial}\left(\frac{\partial}{\partial t} \phi(t)\right) .
$$


Rearranging terms and using (2.4) yields

$$
\begin{aligned}
\sqrt{-1} \partial \bar{\partial}\left(\frac{\partial}{\partial t} \phi(t)\right) & =\sqrt{-1} \partial \bar{\partial}\left(\log \operatorname{det}(g(t))-\log \operatorname{det}\left(g_{0}\right)\right) \\
& =\sqrt{-1} \partial \bar{\partial} \log \frac{\left(\alpha_{t}+\sqrt{-1} \partial \bar{\partial} \phi(t)\right)^{n}}{\omega_{0}^{n}}
\end{aligned}
$$

Therefore the Chern-Ricci flow is equivalent to the scalar complex Monge-Ampère equation

$$
\frac{\partial}{\partial t} \phi=\log \frac{\left(\alpha_{t}+\sqrt{-1} \partial \bar{\partial} \phi(t)\right)^{n}}{\omega_{0}^{n}}, \text { with }\left.\phi\right|_{t=0}=0 .
$$

The proof of Theorem 1.1 will repeatedly exploit the fact that the Chern-Ricci flow (1.1) is equivalent to the scalar flow (2.6).

\section{Proof of Theorem 1.1}

In this section we show that finite-time singularities of the Chern-Ricci flow occur if and only if the scalar curvature is unbounded as $t$ approaches the singular time.

Proof. We will proceed in the same fashion as Zhang in [1] (cf. Section 7.2 of [5]). Let $\omega(t)$ be a solution of the Chern-Ricci flow (1.1) on the maximal interval $[0, T)$ with $T<\infty$. Suppose, by way of contradiction, that there exists a constant $C$ such that

$$
\limsup _{t \rightarrow T}\left(\sup _{M} R(g(t))\right) \leq C .
$$

Throughout this section $C$ will denote a constant which is independent of time, however this constant may change from line to line. We will show that if (3.1) holds, then for any $k \geq 0$ we can produce uniform $C^{k}$ bounds on the metric $\omega(t)$ and hence $T$ is not maximal. We begin by obtaining uniform $C^{0}$ estimates on $\omega(t)$ for $t \in[0, T)$.

First, by studying the evolution of the scalar curvature $R(t)$ under (1.1), we will show that the $C^{0}$ norm of $R(t)$ is uniformly bounded on $[0, T)$.

Lemma 3.1. Under the Chern-Ricci flow (1.1) the scalar curvature of the Chern connection evolves by

$$
\frac{\partial}{\partial t} R=\Delta R+\left|\operatorname{Ric}^{C}(\omega)\right|^{2}
$$

Proof. In local holomorphic coordinates, at a point $p \in M$, we compute

$$
\begin{aligned}
\frac{\partial}{\partial t} R & =\frac{\partial}{\partial t}\left(g^{i \bar{j}} R_{i \bar{j}}^{C}\right) \\
& =g^{k \bar{j}} g^{i \bar{p}} R_{k \bar{p}}^{C} R_{i \bar{j}}^{C}-g^{i \bar{j}} \partial_{i} \partial_{\bar{j}}\left(g^{k \bar{l}} \frac{\partial}{\partial t} g_{k \bar{l}}\right) \\
& =\Delta R+\left|\operatorname{Ric}^{C}(\omega)\right|^{2} .
\end{aligned}
$$

Hence by Lemma 3.1 and the maximum principle, it follows that $R(t)$ is uniformly bounded below on $[0, T)$. Combining this lower bound with (3.1), we have that $\|R(t)\|_{C^{0}}$ is uniformly bounded on $[0, T)$. 
Next we will use the bounds on the scalar curvature $R(t)$ to obtain bounds on the determinant of $\omega(t)$, which are independent of $t$. Recall from (2.6) that the ChernRicci flow is equivalent to the complex Monge-Ampère equation $\frac{\partial}{\partial t} \phi=\log \left(\frac{\omega^{n}}{\omega_{0}^{n}}\right)$. Now, since $\frac{\partial}{\partial t} \log \left(\omega^{n}\right)=-R$, we have

$$
\frac{\partial}{\partial t} \dot{\phi}=-R
$$

Consequently,

$$
\left|\frac{\partial}{\partial t} \dot{\phi}\right|=|R| \leq C
$$

Integrating (3.3) in time we have that there exists a constant $C$ such that

$$
\left|\log \left(\frac{\omega^{n}}{\omega_{0}^{n}}\right)\right|=\left|\frac{\partial}{\partial t} \phi\right| \leq C .
$$

Notice that (3.4) gives uniform bounds on the determinant of $\omega(t)$ on $[0, T)$. Integrating in time again gives uniform bounds for $|\varphi|$. Given uniform estimates on the determinant of the metric, to prove that $\omega(t)$ is uniformly bounded in $C^{0}$, it suffices to show that the trace of the metric $\operatorname{tr}_{g_{0}} g$ is bounded above.

For a bound on the trace of the metric, we repeat the estimates derived by Tosatti-Weinkove in Section 4 of [8] with minor modifications. First let

$$
Q_{1} \doteq t \dot{\phi}-\phi-n t
$$

and notice that $Q_{1}$ is uniformly bounded. Combining the trace of (2.5) with (3.2), it follows that

$$
\left(\frac{\partial}{\partial t}-\Delta\right) \dot{\phi}=\operatorname{tr}_{\omega}\left(-\operatorname{Ric}^{C}\left(\omega_{0}\right)\right)
$$

Taking the trace of (2.4), we have $\Delta \phi=\operatorname{tr}_{\omega}\left(t \operatorname{Ric}^{C}\left(\omega_{0}\right)-\omega_{0}\right)+n$ and so,

$$
\left(\frac{\partial}{\partial t}-\Delta\right) Q_{1}=-\operatorname{tr}_{\omega} \omega_{0}
$$

Let $\widetilde{C}$ denote a constant which is sufficiently large so that $\phi+\widetilde{C} \geq 1$ and let $B$ be a large constant to be chosen below. Next, define

$$
Q_{2} \doteq \log \operatorname{tr}_{g_{0}} g-\phi+\frac{1}{\phi+\widetilde{C}}+B Q_{1} .
$$

Notice that each term in $Q_{2}$, with the possible exception of $\log \operatorname{tr}_{g_{0}} g$, is uniformly bounded. Therefore it is enough to show that $Q_{2}$ is bounded above at a point where $Q_{2}$ achieves its maximum.

By Proposition 3.1 in [8], we have the following estimate on the heat operator applied to $\log \operatorname{tr}_{g_{0}} g$.

Proposition 3.2 (Tosatti-Weinkove [8]). Given a solution $g(t)$ of the Chern-Ricci flow (1.1),

$$
\left(\frac{\partial}{\partial t}-\Delta\right) \log \operatorname{tr}_{g_{0}} g \leq \frac{2}{\left(\operatorname{tr}_{g_{0}} g\right)^{2}} R e\left(g^{\bar{l} k}\left(T_{0}\right)_{k p}^{p} \partial_{\bar{l}} \operatorname{tr}_{g_{0}} g\right)+C \operatorname{tr}_{g} g_{0} .
$$


Now we may assume that at a maximum of $Q_{2}$ that $\operatorname{tr}_{g_{0}} g \geq 1$ since otherwise we have the desired upper bound. For a bound on the first term in (3.8), notice that at a maximum of $Q_{2}, \partial_{i} Q_{2}=0$. Equivalently,

$$
\frac{1}{\operatorname{tr}_{g_{0}} g} \partial_{i} \operatorname{tr}_{g_{0}} g-\partial_{i} \phi-\frac{1}{(\phi+\widetilde{C})^{2}} \partial_{i} \phi=0 .
$$

Hence at a maximum of $Q_{2}$,

$$
\begin{aligned}
& \left|\frac{2}{\left(\operatorname{tr}_{g_{0}} g\right)^{2}} \operatorname{Re}\left(g^{\bar{l} k}\left(T_{0}\right)_{k p}^{p} \partial_{\bar{l}} \operatorname{tr}_{g_{0}} g\right)\right| \\
& \leq\left|\frac{2}{\operatorname{tr}_{g_{0}} g} \operatorname{Re}\left(\left(1+\frac{1}{(\phi+\widetilde{C})^{2}}\right) g^{\bar{l} k}\left(T_{0}\right)_{k p}^{p}\left(\partial_{\bar{l}} \phi\right)\right)\right| \\
& \leq \frac{|\partial \phi|_{g}^{2}}{(\phi+\widetilde{C})^{3}}+C(\phi+\widetilde{C})^{3} \frac{\operatorname{tr}_{g} g_{0}}{\left(\operatorname{tr}_{g_{0}} g\right)^{2}} .
\end{aligned}
$$

Notice that we may also assume that at a maximum of $Q_{2},\left(\operatorname{tr}_{g_{0}} g\right)^{2} \geq(\phi+\widetilde{C})^{3}$ since if this is not the case then we again have the desired upper bound. And so, by (3.6), (3.8), and (3.10), at a maximum

$$
\begin{aligned}
0 \leq & \left(\frac{\partial}{\partial t}-\Delta\right) Q_{2} \\
\leq & \frac{|\partial \phi|_{g}^{2}}{(\phi+\widetilde{C})^{3}}+C \operatorname{tr}_{g} g_{0}-\left(1+\frac{1}{(\phi+\widetilde{C})^{2}}\right) \dot{\phi}+\left(1+\frac{1}{(\phi+\widetilde{C})^{2}}\right) \operatorname{tr}_{g}\left(g-\alpha_{t}\right) \\
& -\frac{2}{(\phi+\widetilde{C})^{3}}|\partial \phi|_{g}^{2}-B \operatorname{tr}_{g} g_{0} \\
\leq & (C-B) \operatorname{tr}_{g} g_{0}+C^{\prime}
\end{aligned}
$$

where $C^{\prime}$ comes from the bounds on $\phi$ and $\dot{\phi}$. Also notice that we used the bound

$$
\begin{aligned}
-\operatorname{tr}_{g} \alpha_{t} & =-\operatorname{tr}_{g} g_{0}+t \operatorname{tr}_{g}\left(\operatorname{Ric}^{C}\left(g_{0}\right)\right) \\
& \leq-\operatorname{tr}_{g} g_{0}+\hat{C} \operatorname{tr}_{g} g_{0}
\end{aligned}
$$

where $\hat{C}$ is a constant that depends on the geometry of $g_{0}$ and harmlessly on $t$. Now we choose $B=C+1$. Hence

$$
\operatorname{tr}_{g} g_{0} \leq C^{\prime}
$$

Finally, using the bounds on the determinant of the metric (3.4) and (3.12),

$$
\operatorname{tr}_{g_{0}} g \leq \frac{1}{(n-1) !}\left(\operatorname{tr}_{g} g_{0}\right)^{n-1} \frac{\operatorname{det} g}{\operatorname{det} g_{0}} \leq C^{\prime \prime}
$$

Thus we have shown that $Q_{2}$ is uniformly bounded above and so $\operatorname{tr}_{g_{0}} g$ is bounded above. This proves the uniform $C^{0}$ bounds on $\omega(t)$; hence there exists a constant $C$ so that

$$
\frac{1}{C} \omega_{0} \leq \omega(t) \leq C \omega_{0}
$$

for $t \in[0, T)$.

The higher order estimates on the metric follow from the first-named author in 2]. This proves the theorem. 


\section{Proof of Theorem 1.2}

The proof follows rather quickly from the previous proof and the results of Tosatti-Weinkove and follows a method similar to that in [1]. In the case of finitetime non-collapsing, Tosatti-Weinkove have shown that there exist finitely many disjoint $(-1)$ curves $E_{1}, \ldots, E_{l}$ on $M$ with $\operatorname{Vol}\left(E_{i}, g(t)\right) \rightarrow 0$ as $t \rightarrow T$ [8]. This immediately implies that $E \subset \Sigma$. Tosatti-Weinkove also proved that the flow converges smoothly on compact sets away from $E$, giving the reverse containment since no singularities develop outside of $E$ and hence $E=\Sigma[10$.

Trivially, we have $\Sigma^{\prime} \subset \Sigma=E$. It remains to show that for any $x$ in $M$ with an open neighborhood $U$ on which $|R(t)| \leq C$ for all $0 \leq t<T$, we have smooth convergence on a smaller open neighborhood $U^{\prime}$ of $x$. A key observation is that most of the estimates from the proof of Theorem 1.1 are in fact local and therefore will be applied again here.

First let $K$ be a compact subset of $U$ and let $U^{\prime}$ be an open neighborhood of $x$ contained in $K$. We will derive uniform $C^{0}$ estimates on $\omega(t)$ in $K$ and thus on $U^{\prime}$. Since $|R(t)|$ is bounded on $U$, it follows from (3.3) and (3.4) that the determinant of $\omega(t)$ is bounded on $K \times[0, T)$. To show that $\omega(t)$ is bounded in $C^{0}$ on $K \times[0, T)$, it is enough to show that $\operatorname{tr}_{g_{0}} g$ is bounded above on this set.

Let $Q_{1}$ be defined as above in (3.5) and let

$$
Q_{2}=\log \operatorname{tr}_{g_{0}} g-\phi+\frac{1}{\phi+\widetilde{C}}+B Q_{1}
$$

as in (3.7). Since $|R(t)|$ is uniformly bounded on $K$, we again have that each term in $Q_{2}$, except possibly $\log \operatorname{tr}_{g_{0}} g$, is uniformly bounded. It remains to show that $Q_{2}$ is bounded above at a point where $Q_{2}$ achieves its maximum in $K$.

For the term $\log \operatorname{tr}_{g_{0}} g$ in $Q_{2}$, we have that

$$
\left(\frac{\partial}{\partial t}-\Delta\right) \log \operatorname{tr}_{g_{0}} g \leq \frac{2}{\left(\operatorname{tr}_{g_{0}} g\right)^{2}} R e\left(g^{\bar{l} k}\left(T_{0}\right)_{k p}^{p} \partial_{\bar{l}} \operatorname{tr}_{g_{0}} g\right)+C \operatorname{tr}_{g} g_{0}
$$

by Proposition 3.1 in [8]. Furthermore at a maximum of $Q_{2}$ in $K$, from (3.10) we have

$$
\left|\frac{2}{\left(\operatorname{tr}_{g_{0}} g\right)^{2}} \operatorname{Re}\left(g^{\bar{l} k}\left(T_{0}\right)_{k p}^{p} \partial_{\bar{l}} \operatorname{tr}_{g_{0}} g\right)\right| \leq \frac{|\partial \phi|_{g}^{2}}{(\phi+\widetilde{C})^{3}}+C(\phi+\widetilde{C})^{3} \frac{\operatorname{tr}_{g} g_{0}}{\left(\operatorname{tr}_{g_{0}} g\right)^{2}}
$$

Combining the two estimates above with (3.6) yields

$$
0 \leq\left(\frac{\partial}{\partial t}-\Delta\right) Q_{2} \leq(C-B) \operatorname{tr}_{g} g_{0}+C^{\prime}
$$

see (3.11). So by choosing $B$ sufficiently large, in particular $B=C+1$, it follows that

$$
\operatorname{tr}_{g} g_{0} \leq C^{\prime}
$$

at a maximum in $K \times[0, T)$. And so, as in (3.13), there exists a constant $C^{\prime \prime}$ so that

$$
\operatorname{tr}_{g_{0}} g \leq C^{\prime \prime}
$$

This proves that $Q_{2}$, and thus $\operatorname{tr}_{g_{0}} g$, is bounded above on $K \times[0, T)$. Finally, since $U^{\prime} \subset K$, we have local $C^{0}$ estimates on $\omega(t)$ in $U^{\prime} \times[0, T)$. The local higher order estimates follow by work of Sherman-Weinkove [4]. 


\section{The Hopf MANIFOLD}

Let $\alpha=\left(\alpha_{1}, \ldots, \alpha_{n}\right) \in \mathbb{C}^{n} \backslash\{0\}$ with $\left|\alpha_{1}\right|=\ldots=\left|\alpha_{n}\right| \neq 1$. Then consider the Hopf manifold $M_{\alpha}=(\mathbb{C} \backslash\{0\}) / \sim$ where $\left(z_{1}, \ldots, z_{n}\right) \sim\left(\alpha_{1} z_{1}, \ldots, \alpha_{n} z_{n}\right)$. Let

$$
\begin{aligned}
& \omega_{H}=\frac{\delta_{i \bar{j}}}{r^{2}} \sqrt{-1} d z^{i} \wedge d z^{\bar{j}}, \\
& \operatorname{Ric}^{C}\left(\omega_{H}\right)=\frac{n}{r^{2}}\left(\delta_{i \bar{j}}-\frac{z_{i} z_{\bar{j}}}{r^{2}}\right) \sqrt{-1} d z^{i} \wedge d z^{\bar{j}} .
\end{aligned}
$$

Tosatti-Weinkove show that the metric

$$
\omega(t)=\omega_{H}-t \operatorname{Ric}^{C}\left(\omega_{H}\right)=\left(\frac{\delta_{i \bar{j}}}{r^{2}}-\frac{n t}{r^{2}}\left(\delta_{i \bar{j}}-\frac{z_{i} z_{\bar{j}}}{r^{2}}\right)\right) \sqrt{-1} d z^{i} \wedge d z^{\bar{j}}
$$

is a solution to the Chern-Ricci flow on the Hopf manifold [8]. In particular, they show that $\operatorname{Ric}^{C}(\omega(t))=\operatorname{Ric}^{C}\left(\omega_{H}\right)$.

Computing directly, we see that the Chern scalar curvature blows up at $T=\frac{1}{n}$ and additionally

$$
R(\omega(t))=\operatorname{tr}_{\omega(t)} \operatorname{Ric}^{C}\left(\omega_{H}\right) \leq \frac{C}{\frac{1}{n}-t}
$$

\section{ACKNOWLEDGMENTS}

The authors would like to especially thank Valentino Tosatti for pointing out that Theorem 1.2 should follow from the proof of Theorem 1.1. The second-named author would also like to thank Jon Wolfson for numerous helpful conversations. The authors would also like to thank Ben Weinkove and the referee for their useful comments.

\section{REFERENCES}

[1] T. Collins and V. Tosatti, Kähler currents and null loci, ArXiv e-prints (2013).

[2] Matt Gill, Convergence of the parabolic complex Monge-Ampère equation on compact Hermitian manifolds, Comm. Anal. Geom. 19 (2011), no. 2, 277-303, DOI 10.4310/CAG.2011.v19.n2.a2. MR.2835881 (2012h:32047)

[3] Ke-Feng Liu and Xiao-Kui Yang, Geometry of Hermitian manifolds, Internat. J. Math. 23 (2012), no. 6, 1250055, 40, DOI 10.1142/S0129167X12500553. MR2925476

[4] Morgan Sherman and Ben Weinkove, Local Calabi and curvature estimates for the ChernRicci flow, New York J. Math. 19 (2013), 565-582. MR3119098

[5] Jian Song and Ben Weinkove, An introduction to the Kähler-Ricci flow, An introduction to the Kähler-Ricci flow, Lecture Notes in Math., vol. 2086, Springer, Cham, 2013, pp. 89-188, DOI 10.1007/978-3-319-00819-6_3. MR.3185333

[6] Jeffrey Streets and Gang Tian, A parabolic flow of pluriclosed metrics, Int. Math. Res. Not. IMRN 16 (2010), 3101-3133, DOI 10.1093/imrn/rnp237. MR2673720 (2011h:53091)

[7] Jeffrey Streets and Gang Tian, Hermitian curvature flow, J. Eur. Math. Soc. (JEMS) 13 (2011), no. 3, 601-634, DOI 10.4171/JEMS/262. MR2781927 (2012f:53142)

[8] Valentino Tosatti and Ben Weinkove, On the evolution of a Hermitian metric by its ChernRicci form, J. Differential Geom. 99 (2015), no. 1, 125-163. MR.3299824

[9] V. Tosatti, B. Weinkove, and X. Yang, Collapsing of the Chern-Ricci flow on elliptic surfaces, ArXiv e-prints (2013).

[10] Valentino Tosatti and Ben Weinkove, The Chern-Ricci flow on complex surfaces, Compos. Math. 149 (2013), no. 12, 2101-2138, DOI 10.1112/S0010437X13007471. MR3143707 
[11] Zhou Zhang, Scalar curvature behavior for finite-time singularity of Kähler-Ricci flow, Michigan Math. J. 59 (2010), no. 2, 419-433, DOI 10.1307/mmj/1281531465. MR.2677630 (2011j:53128)

Department of Mathematics, University of California, Berkeley, 970 Evans Hall \#3840, Berkeley, California 94720-3840

Department of Mathematics, Furman University, 3300 Poinsett Highway, Greenville, South Carolina 29613 